\title{
Interference Cancellation for the Coexistence of 5.8 GHz DSRC and 5.9 GHz ETSI ITS
}

\author{
Stefano Maddio, Alessandro Cidronali, Marco Passafiume, Giovanni Collodi, Gianfranco Manes \\ Department of Information Engineering \\ University of Florence, V. S. Marta, 3, I-50139, Florence, Italy \\ e-mail: \{name.surname\}@unifi.it
}

\begin{abstract}
In this paper we analyze the cancellation of the interference caused by a Intelligent Transportation Systems (ITS) on devices operating in the Dedicated Short Range Communications (DSRC) framework. The cancellation is operated by an interference canceller based on the active feed-forward architecture. The canceller is designed to operate over the frequency band $5.2 \mathrm{GHz}-6.4 \mathrm{GHz}$, hence it is suitable for the mitigation of mutual interference on signal pertinent to DSRC at $5.8 \mathrm{GHz}$ due to ITS signals at 5.9 GHz. When applied to a Road Side Unit (RSU) for electronic toll collection (ETC) operating at $5.8 \mathrm{GHz}$, the proposed technique is capable to improve the performance of the front-end by cancelling the interfering signal in the $5.9 \mathrm{GHz}$ bandwidth such as IEEE 802.11p signals involved in the ITS protocols; signal-to-interference improvement of $25 \mathrm{~dB}$ operating on $10 \mathrm{MHz}$ bandwidth signal is herein reported. The paper introduces the architecture of the canceller as well as the experimental results which describe the capability of the technical approach.
\end{abstract} ITS

Keywords-Interference mitigation, analog cancellation, DSRC,

\section{INTRODUCTION}

In the framework of Intelligent Transportation System (ITS), we are assisting to the convergence of various communication platforms in the same physical equipment. Emerging applications need to coexist with existing services, such as vehicular Electronic Toll Collection (ETC) applications. For the latter, the European Telecommunications Standards Institute (ETSI) technical committee recommends Dedicated Short Range (DSRC) Communication in the $5.8 \mathrm{GHz}$ band,[1].

The ITS framework was recently regulated in the same frequency band of ETC, with channels allocated around $5.9 \mathrm{GHz}$. European DSRC equipments, which operate in the frequency range from $5795 \mathrm{MHz}$ to $5815 \mathrm{MHz}$, might suffer from severe interference issues unless same interference cancellation techniques is applied to the device receiver. The difficulties of the ITS-DSRC coexistence are caused by characteristic of the radio link between the Road-Side Unit (RSU) and the OnBoard Unit (OBU) for ETC applications. The OBU is a semipassive RFID-like device, hence it is not equipped with a local oscillator [2], [3]. Thus, upon the reception of the $\mathrm{CW}$ signal from the RSU, it over-imposes its payload by a back-scattering technique; in this process the carrier is ASK modulated with a BPSK modulated sub-carrier.

Considering that the RSU maximum Effective Isotropic Radiated Power (EIRP) is limited to $33 \mathrm{dBm}$, and that common range of 7 meters with antenna gain around $12 \mathrm{dBi}$ are involved, the received DSRC signal is expected in the $90 \mathrm{dBm}$ to $-80 \mathrm{dBm}$ range. On the other side, the ITS signals are based on IEEE $802.11 \mathrm{p}$ protocol, with $10 \mathrm{MHz}$ OFDM channel operating with EIRP of $33 \mathrm{dBm}$; according with the various scenarios defined in [4], the blocking signal at the DSRC RSU are expected between $-60 \mathrm{dBm}$ to $-20 \mathrm{dBm}$. In the worst case scenario a signal-interference ratio (SIR) of $-50 \mathrm{~dB}$, is expected. The latter issue translates in severe saturation problem of the first active stage of the RSU receiver.

This paper deals with the depicted issue in the framework of the RSU operation, discussing a topology for ITS signal rejection in conjunction to a $5.8 \mathrm{GHz}$ receiver for ETC applications, and provides experimental validation of the proposed technique, by developing a prototype which involves commercially available (COTS) subsystems.

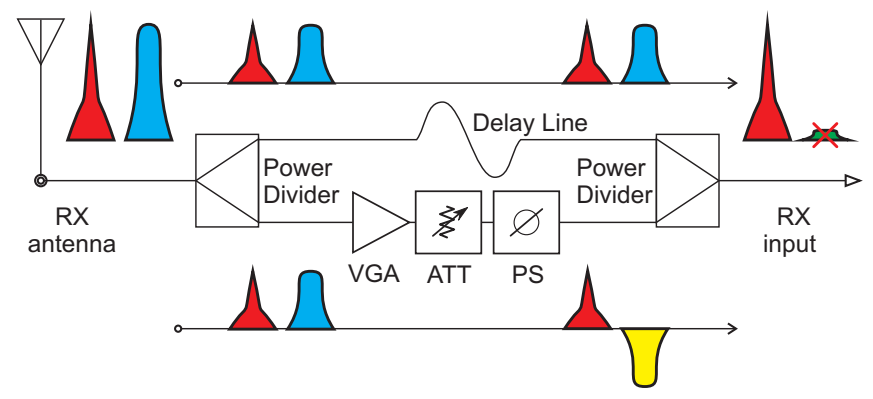

Fig. 1. Schematic diagram of the interference canceller system and definition of the relevant signals.

\section{MeChanism of INTERFERENCE CANCELLATION}

\section{A. Interference canceller topology}

Analog cancellation [5] is based on the feed-forward control of the interference signal (aggressor), whose topology is schematically depicted in Fig. 1, [6], [7], [8]. In the interference canceller system, the signal on the RX path is divided through two paths by a power divider (PD); although there might be convenience in an uneven power split in through this paper we consider a $-3 \mathrm{~dB}$ splitting. One of the signal path introduces a magnitude and phase manipulation to be successively injected again into the receive path by a second PD. The goal is to synthesize the inverted replica of the aggressor (in our case the $5.9 \mathrm{GHz}$ interference) without affecting the victim (the $5.8 \mathrm{GHz}$ DRSC signal).

In the process of suppressing the aggressor signal, an unwanted manipulation on the victim signal is introduced as well. The goal is to maximize the SIR at the output of the canceller. This implies that the canceller has to be 
able to operate autonomously as the aggressor as well as the victim are time-variant signals. The analytical treatment of the cancelling mechanism and its adaptation are hereby described. This treatment is derived from the operation mode described in [9] by the authors.

\section{B. Operating principles of the interference canceller}

Figure 2 shows the phasor representation of the signals involved in the cancelling process. Due to the first PD the original combination of $\mathbf{S}$ and $\mathbf{I}$ is injected in the two parallel paths, hence the label $\mathbf{S}_{\mathbf{U}}$ and $\mathbf{I}_{\mathbf{U}}$ for the upper path and $\mathbf{S}_{\mathbf{D}}$ and $\mathbf{I}_{\mathbf{D}}$ for the corresponding signals in the lower path. Focusing on the interference, by the means of the cascade of a variable gain amplifier (VGA in Fig. 1) and a phase shifter (PS), with the addition of a controlled attenuator (ATT) for the fine tuning, the $\mathbf{I}_{\mathbf{D}}$ is manipulated to invert its phase.

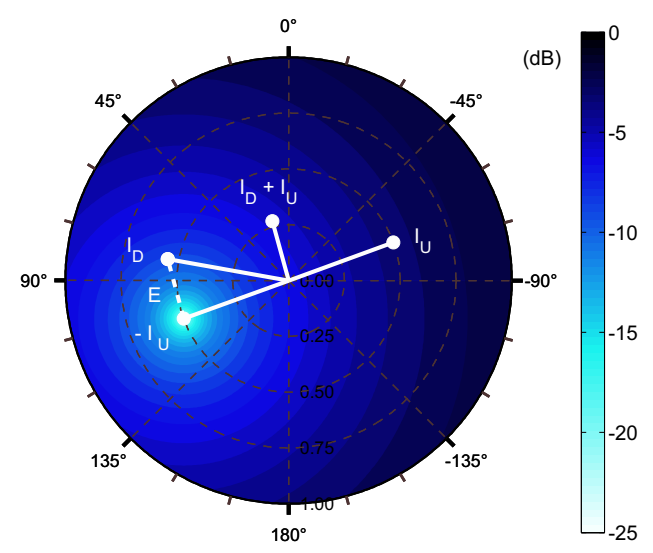

(a) Cancellation of the interference $\mathbf{I}$ pursuing $\mathbf{I}_{\mathbf{D}}=-\mathbf{I}_{\mathbf{U}}$

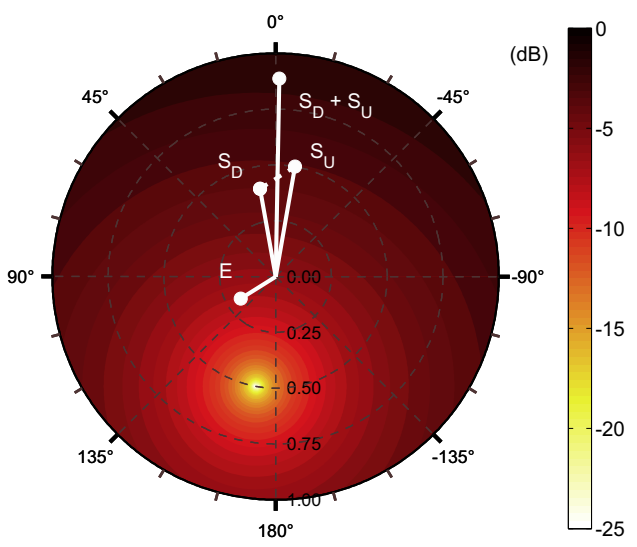

(b) Preservation of the victim signal $\mathbf{S}$ pursuing $\mathbf{S}_{\mathbf{D}}=\mathbf{S}_{\mathbf{U}}$

Fig. 2. Phasor representation of the signals involved in the interference mitigation.

The signal to be re-introduced in the combiner should be $\mathbf{I}_{\mathbf{D}}=-\mathbf{I}_{\mathbf{U}}$. The signal $\mathbf{E}=\mathbf{I}_{\mathbf{U}}+\mathbf{I}_{\mathbf{D}}$ is therefore an error signal, measuring the distance to the actual $\mathbf{I}_{\mathbf{D}}$ to the goal antiinterference signal $-\mathbf{I}_{\mathbf{D}}$. By the above definition, it is worth noting that the magnitude of $\mathbf{E}$ can be written as, [9],

$$
|\mathbf{E}|^{2}=\left|\mathbf{I}_{\mathbf{U}}\right|^{2}+\left|\mathbf{I}_{\mathbf{D}}\right|^{2}-2\left|\mathbf{I}_{\mathbf{U}}\right|\left|\mathbf{I}_{\mathbf{D}}\right| \cos \left(\pi-\left(\phi_{I_{U}}-\phi_{I_{D}}\right)\right)
$$

Defining the magnitude and phase unbalance of $\mathbf{I}_{\mathbf{U}}$ and $\mathbf{I}_{\mathbf{D}}$ as $A=\left|\mathbf{I}_{\mathbf{U}}\right| /\left|\mathbf{I}_{\mathbf{D}}\right|$ and $\delta=\left(\phi_{I_{U}}-\phi_{I_{D}}\right)$ we can rewrite the relative error function as

$$
E=E(A, \delta)=\left|\mathbf{I}_{\mathbf{U}}\right| \sqrt{1+A^{2}+2 A \cos (\delta)}
$$

The above expression is therefore a closed-form function the PS phase $\phi$ and ATT attenuation $A$, and it is minimised for $A=1$ and $\delta=\pi$, when the perfect interference cancellation is obtained, as depicted in Fig. 2a.

The same signal manipulation mechanism is necessarily applied to $\mathbf{S}_{\mathbf{U}}$ and $\mathbf{S}_{\mathbf{U}}$, with the difference that the final expression, which is formally identical to (2), has to be maximized to preserve the signal of interest, therefore, the condition to be pursued is $A=1$ and $\delta=0$, - see Fig. 2b. In conclusion, the conditions $\delta=0$ and $\delta=\pi$ have to be imposed operating on the same canceling signal, i.e. the same ATT and PS controls. In order to accomplish this opposite behavior at the two frequency of signal and aggressor, the device should provide different phase lag to the two frequencies at which signal and interference are located. The delay line in the upper branch depicted in Fig. 1 provides the necessary phase difference in the most straight-forward way.

According to the specific characteristics of the input stage of the transceiver (1dBP, IMD3 etc.), the best improvement of the SIR could occur even if the interference is not completely nullified. In fact, it is enough to have a strong mitigation of interference if at the same time the signal of interest is preserved in its integrity and possibly enhanced. Indeed, due to amplification within the lower branch in Fig. 1, the signal can be even slightly amplified at the output of the device.

\section{PROTOTYPE CHARACTERIZATION}

To better clarify the design of the proposed canceller a prototype was developed based on COTS subsystems. The prototype is composed of two microstrip power splitters and combiners at the end of the two parallel paths. The principal path is composed of an RF amplifier (HMC717) in cascade with a 6-bit digital phase shifter LSB of 5.6 degrees (HMC649LP6), and a 6-bit digital attenuator $0.5 \mathrm{~dB}$ LSB (HMC425LP3). The combination of amplification and attenuation compensates the loss of the phase shifter and the subsystems insertion losses, while provides a prototype flexibility. The secondary path consists simply in a delay line, characterized by a proper group delay. In particular, the line length is chosen to exhibit $1.8 \mu \mathrm{s}$ group delay. In this way, the phase shown at a fixed frequency $f_{1}$ is the opposite of the phase exhibited at $f_{2}=f_{1}+100 \mathrm{MHz}$. Figure 3 shows the phase difference due to the different paths of the upper and lower branch of the canceller. The measured slope equal the optimal goal of $1.8^{\circ}$ per $\mathrm{MHz}$ and it preserves through the entire range of the controllable phase shifter.

Figure 4 reports the transfer function response versus frequency for the prototype when it is set for the rejection of signal in the $5900 \mathrm{MHz}$ band. The sharp notch-like shape leaves the band of the signal of interest slightly affected, while the rejection at the central frequency of $5900 \mathrm{MHz}$ exceeds $30 \mathrm{~dB}$ of isolation and at least $20 \mathrm{~dB}$ is obtained within a $10 \mathrm{MHz}$ bandwidth around it. Periodical presence of zeros confirms the resonant behavior of the phase difference as depicted in Fig. 3. 


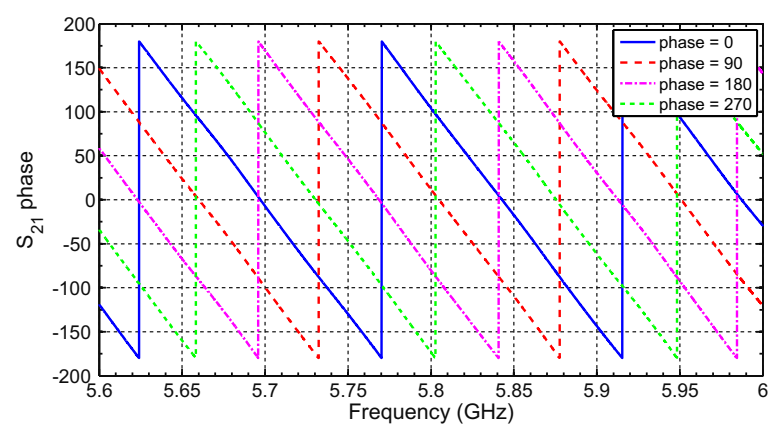

Fig. 3. Phase difference of the upper and lower branches of the canceller.

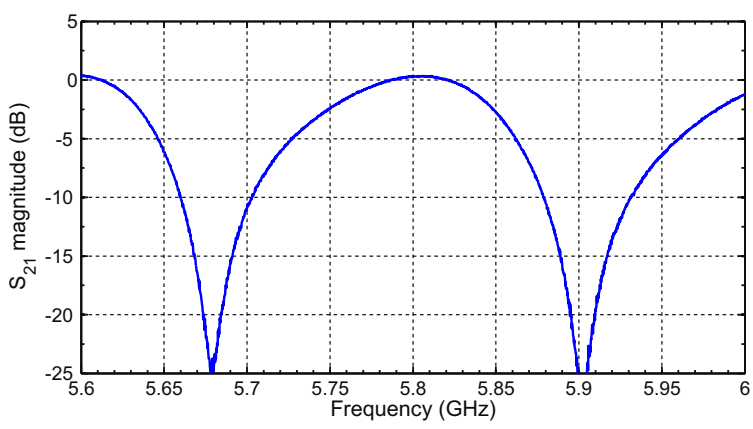

Fig. 4. Transfer function for the cancellation of the $5900 \mathrm{MHz}$ channel.

\section{EXPERIMENTAL VALIDATION}

The exhaustive matrices of cases depicted in Fig. 5 are obtained setting the variable gain from $-2 \mathrm{~dB}$ to $+8 \mathrm{~dB}$ and varying the PS phase over the range of $360^{\circ}$. In particular Fig. 5b, Fig. 5c and Fig. 5d, depict the measured magnitude of the interference signal located at $5880 \mathrm{MHz}, 5890 \mathrm{MHz}$ and $5900 \mathrm{MHz}$, while Fig. 5a depicts the magnitude of DSRC signal in the same conditions. From direct inspection is confirmed that the conditions mitigating the aggressors are almost in antiphase with the conditions which are disruptive for the signal of interest. therefore, setting the PS and VGA+ATT controls to match this conditions results in a net SIR improvement.

In a real-time application, the canceller setting has to dynamically change according to the variations of the ITS signals, as the OFDM channel changes according to $802.11 \mathrm{p}$ protocol. Differently from solutions found in literature [8], [10], the reconfiguration of the proposed device can be autonomous, based on 5-steps algorithms, described in [9]. The key of the reconfiguration is the closed-form model expressed in (2), which permits the feedback on the controls on the base of the scalar measurement of the signal magnitude. Thanks to the automatic reconfiguration the proposed canceller can even track environmental variations, such as the fast variation of radio-wave scattering due to vehicular flow, as well as slow variations for aging and temperature fluctuation. As long as the variation is inside the domain of the canceller, i.e. it can be matched with the gain variation, the cancellation can be stably achieved.

Figure 6 shows the spectrum of the signals at the input port of the receiver. In particular, Fig. 6a depicts the situation withour the interference canceller, while Fig. 6b shows the case

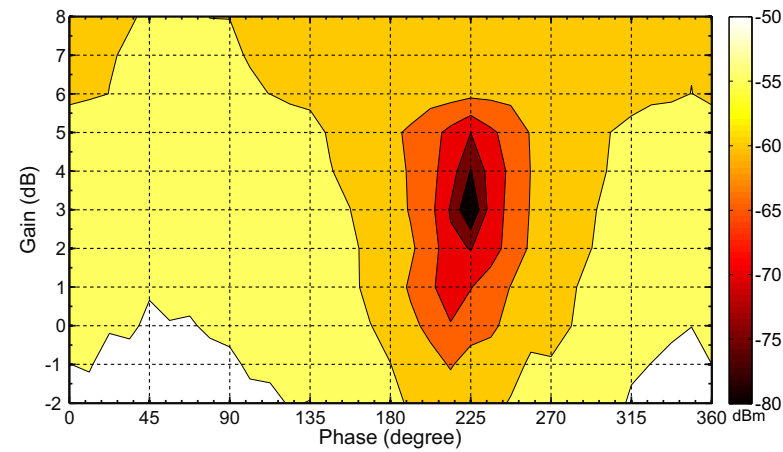

(a) Signal at 5800 for the matrix of cases

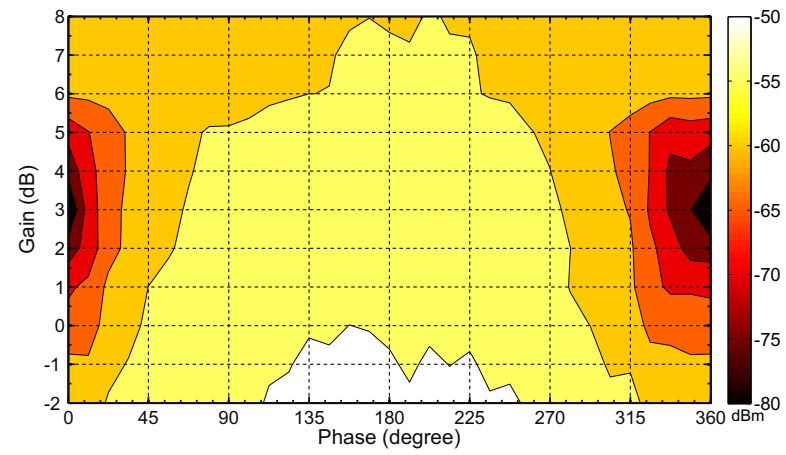

(b) Interference at $5880 \mathrm{MHz}$ for the matrix of cases

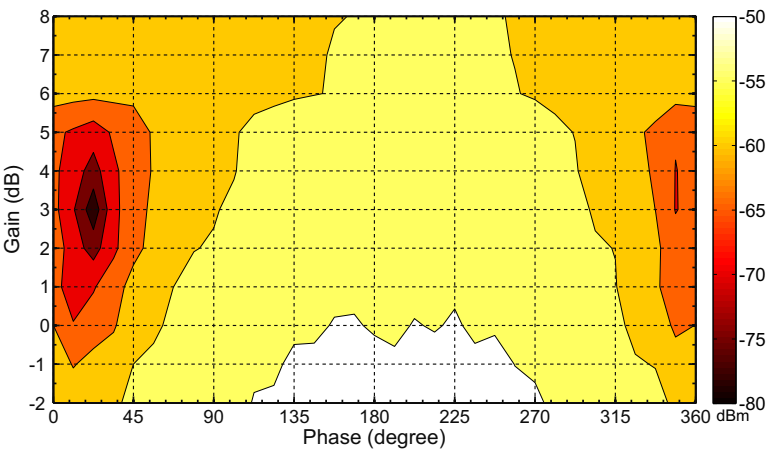

(c) Interference at $5890 \mathrm{MHz}$ for the matrix of cases

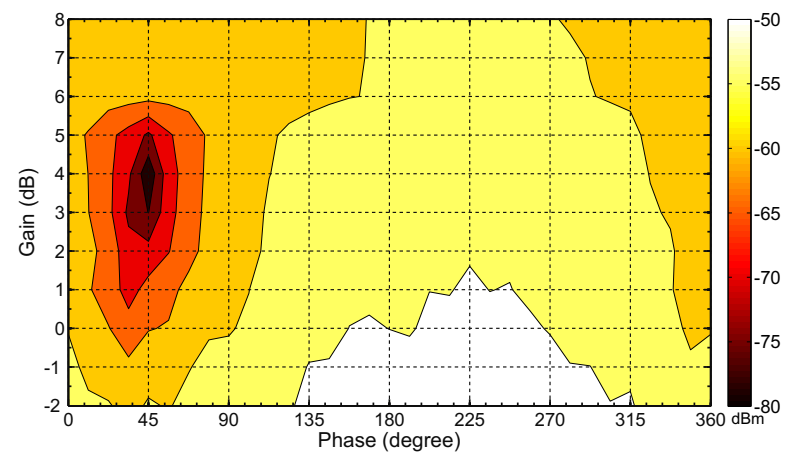

(d) Interference at $5900 \mathrm{MHz}$ for the matrix of cases

Fig. 5. Magnitude of the signals of interest. In abscissa the phase $\phi_{P S}$ of the PS, in ordinate the gain $G$ of the VGA+ATT subsystem. The phase of the minima for the three ITS interference signal is between $\phi_{P S}=0$ in (d) and $\phi_{P S}=45$ in (b). This corresponds to plateau around the maximum for the DSRC signal in (a), which happens with the same Gain control. 


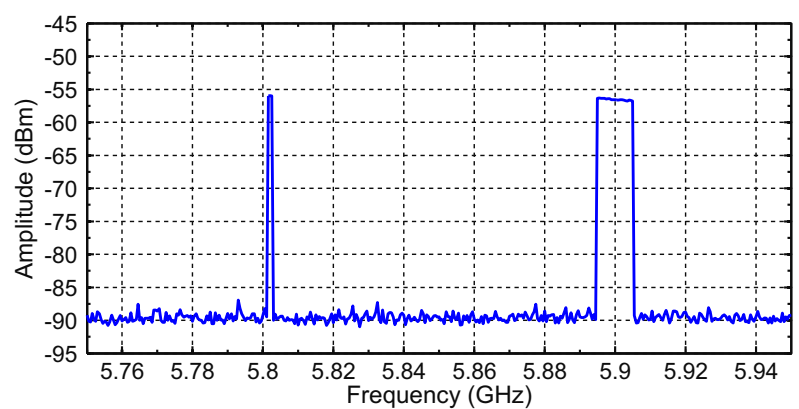

(a) Without the interference canceller

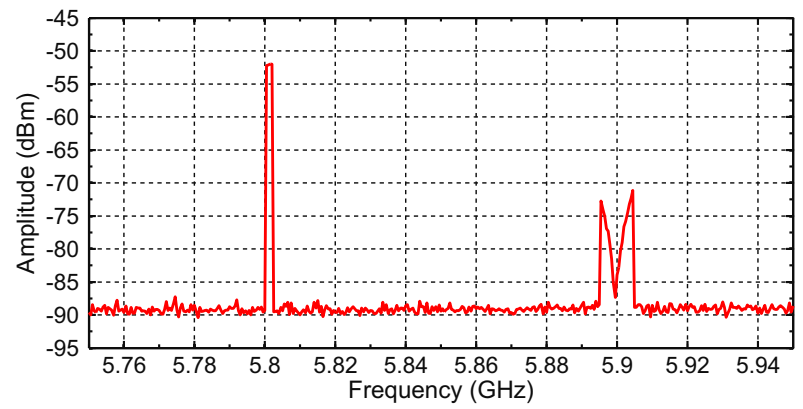

(b) With the interference canceller

Fig. 6. Spectrum of DSRC signal at $5.8 \mathrm{GHz}$ and ITS signal at $5.9 \mathrm{GHz}$ with (b) and without (a) the interference canceller.

with the canceller self-adjusted for the cancellation. While the cancellation is exact only at the central frequency - following the shape of the transfer function depicted in Fig. 4 - the entire $10 \mathrm{MHz}$ bandwidth around the $5.9 \mathrm{GHz}$ carrier is attenuated, with minimal impact on the signal of interest at $5.8 \mathrm{GHz}$.

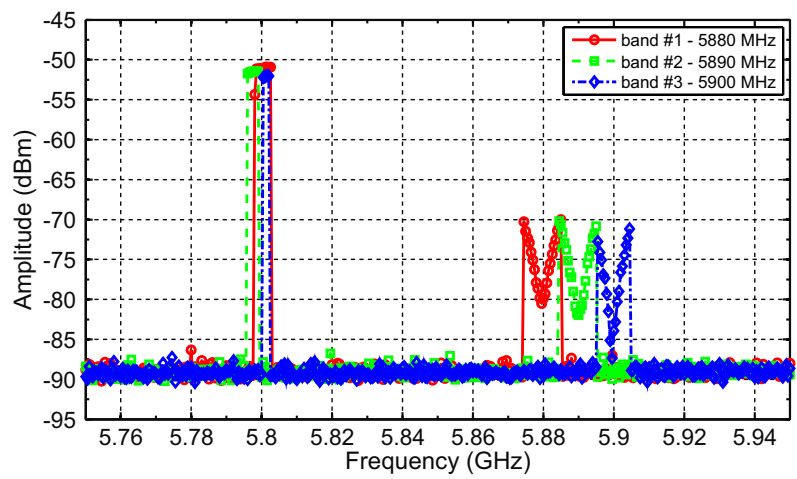

Fig. 7. Cancellation of $10 \mathrm{MHz}$ bandwidth signal centered at three different frequencies.

Figure 7 depicts the effects of the interference canceller on three different ITS signals, meaning three $10 \mathrm{MHz}$ OFDM signals centered at $5.880 \mathrm{GHz}, 5.890$ and $5.900 \mathrm{GHz}$. Being the delay line designed to grant $180^{\circ}$ at $100 \mathrm{MHz}$, the best cancellation is obtained at the frequency of $5.9 \mathrm{GHz}$. Nevertheless, satisfying recombination can be obtained also at $5880 \mathrm{MHz}$ and $5890 \mathrm{MHz}$, even if at the price of a less favorable SIR enhancement. Improved results would be achieved with a more complex delay line based on local negative group delay, in order to maintain the same phase difference experimented by the $5.9 \mathrm{GHz}$ signal even at $5.88 \mathrm{GHz}$.

Nevertheless, the improvement in terms of SIR can be quantified evaluating the SIR before and after the operations over the entire $10 \mathrm{MHz}$ bandwidth of interest for the ITS signal. In the case under consideration, the enhancement of SIR is evaluated as $24 \mathrm{~dB}, 24.4 \mathrm{~dB}$ and $25.8 \mathrm{~dB}$ for the three cases in exam. These performance are consistent with the phase matching of the three bandwidth with respect to the DSRC in Fig. 5 and with the frequency behavior depicted in Fig. 4.

\section{CONCLUSION}

In this paper a interference canceller enabling the coexistence of DSRC at $5.8 \mathrm{GHz}$ with ITS signals at $5.9 \mathrm{GHz}$ was proposed. The interference canceller is based on the feed-forward cancellation principle, manipulating the involved waveform in order to mitigate the undesired interfering signal while enhancing or at least keeping unmodified the signal of interest. The design was verified with a proof-of-concept prototype based on commercial component. Even if sub-optimal, the assembled prototype demonstrate SIR improvement in the order of $25 \mathrm{~dB}$ and adequate reconfigurability to deal with three different operation bands.

\section{REFERENCES}

[1] ES200674-1 V1.2.I, Electromagnetic compatibility and Radio spectrum Matters (ERM); Road Transport and Traffic Telematics (RTTT);Part 1: Technical characteristics and test methods for High Data Rate (HDR) data transmission equipment operating in the 5,8 GHz Industrial, Scientific and Medical (ISM) band, ETSI, 2007.

[2] R. Chakraborty, S. Roy, and V. Jandhyala, "Revisiting RFID Link Budgets for Technology Scaling: Range Maximization of RFID Tags," Microwave Theory and Techniques, IEEE Transactions on, vol. 59, no. 2, pp. 496-503, 2011.

[3] S. Maddio, A. Cidronali, S. Maurri, and G. Manes, "Compact ETSI compliant DSRC transponder for vehicular communications at 5.8 GHz," in Microwave Radar and Wireless Communications (MIKON), 2012 19th International Conference on, vol. 1, May 2012, pp. 350353.

[4] ETSI TR 102960 V1.1.1 Intelligent Transport Systems (ITS); Mitigation techniques to avoid interference between European CEN Dedicated Short Range Communication (RTTT DSRC) equipment and Intelligent Transport Systems (ITS) operating in the $5 \mathrm{GHz}$ frequency range; Evaluation of mitigation methods and techniques, ETSI, 2012.

[5] T. D. Werth, C. Schmits, R. Wunderlich, and S. Heinen, "An active feedback interference cancellation technique for blocker filtering in rf receiver front-ends," IEEE J. Solid-State Circuits, vol. 45, no. 5, pp. 989-997, 2010.

[6] S. Maddio, A. Cidronali, and G. Manes, "Interference active cancellation techniques for agile transceivers," in White Space Communication Technologies, N. B. Carvalho, A. Cidronali, and R. Gomez Garcia, Eds. Cambridge: Cambridge University Press, October 2014, p. 231.

[7] T. Xiong, X. Tan, J. Xi, and H. Min, "High TX-to-RX Isolation in UHF RFID Using Narrowband Leaking Carrier Canceller," IEEE Microw. Compon. Lett., vol. 20, no. 2, pp. 124-126, 2010.

[8] J. Jung, C. Park, and K. Yeom, "A novel carrier leakage suppression front-end for uhf rfid reader," IEEE Trans. Microw. Theory Techn., vol. 60, no. 5, pp. 1468-1477, 2012.

[9] S. Maddio, A. Cidronali, and G. Manes, "Real-Time Adaptive Transmitter Leakage Cancelling in 5.8 GHz Full-Duplex Transceivers," IEEE Trans. on Microwave Theory and Tech., vol. 63, no. 2, pp. 509 - 519, 2015.

[10] T. wen Xiong, X. Tan, J. tian Xi, and H. Min, "High tx-to-rx isolation in uhf rfid using narrowband leaking carrier canceller," IEEE Microw. Compon. Lett., vol. 20, no. 2, pp. 124-126, Feb 2010. 